\title{
An Analytical Comparison of the Principal Component Method and the Mixed Effects Model for Association Studies in the Presence of Cryptic Relatedness and Population Stratification
}

\author{
Kai Wang ${ }^{\mathrm{a}} \quad$ Xijian $\mathrm{Hu}^{\mathrm{b}} \quad$ Yingwei Peng ${ }^{\mathrm{c}}$ \\ ${ }^{a}$ Department of Biostatistics, College of Public Health, University of lowa, lowa City, lowa, USA; ${ }^{b}$ Department \\ of Statistics, College of Mathematics and System Science, Xinjiang University, Urumqi, P.R. China; 'Department \\ of Public Health Sciences, and Department of Mathematics and Statistics, Queen's University, Kingston, Ont., \\ Canada
}

\section{Key Words}

Principal component $\cdot$ Mixed effects model $\cdot$ Population stratification - Cryptic relatedness - Genetic association

\begin{abstract}
The principal component method and the mixed effects model represent two popular approaches to controlling for population structure and cryptic relatedness in genetic association studies. There are only a handful of studies comparing their performance. These studies are typically based on simulation studies and the results are therefore limited in their applicability. In this paper, we conduct an analytical comparison of these two approaches in the presence of cryptic relatedness and population structure in terms of their validity and efficiency. In the presence of cryptic relatedness, we show that both methods are valid, but the mixed effects model is more powerful for detecting association. In the presence of population structure, however, we show that both methods can be invalid. The biases and variances of the estimates from the two methods are compared. Examples and simulation studies are provided to demonstrate the conclusions.
\end{abstract}

(c) 2013 S. Karger AG, Basel

\section{KARGER}

(c) 2013 S. Karger AG, Basel

0001-5652/13/0761-0001\$38.00/0

E-Mail karger@karger.com

www.karger.com/hhe

\section{Introduction}

Population stratification is a well-known confounding factor in genetic association studies. It can lead to spurious association if not dealt with appropriately. It exists even in populations that seem to be homogeneous, for instance, European American [1] and Han Chinese [2, 3]. Nowadays more and more large consortia and collaborations are routinely formed in order to identify genetic factors of small effect. For instance, the GENEVA project [4] involves 14 participating studies covering a wide range of primary phenotypes. Each study consists of thousands of study subjects. It is not surprising that population stratification is a pressing issue to be addressed in analyzing data from large-scale multi-center studies. Indeed, the GENEVA coordinating center has developed its own software package to handle this issue (https://www.genevastudy.org/Accomplishments/software).

The genomic control method [5-7] is a popular method for handling population stratification. It modifies the Cochran-Armitage test for trend by a deflating factor. This factor is estimated using markers that are known to 
be unassociated with the phenotype (null markers). The structured population method $[8,9]$ tries to infer the subpopulation structure first. Subsequent analyses are then conducted within each subpopulation and the results are summarized. The third method is to create surrogates for each population using null markers. For example, the principal component analysis (PCA) method [10] uses the first few principal components of the matrix of relatedness as covariates in a regression analysis. Similarly, one can use the first few components from a partial least-squares regression analysis [11]. Given the huge amount of genome-wide genotype data, it is feasible to estimate the relatedness of study subjects [12-15]. This possibility has resulted in novel approaches to genome-wide association studies. The mixed effects model method $[16,17]$ can take into account fixed effects, such as age and gender, while modeling population structure and cryptic relatedness as random effects. Preliminary studies have found that these methods perform better than methods that do not model relatedness of study subjects [16-18]. Most of these methods have been implemented in various computer programs such as STRUCTURE [19, 20], ADMIXTURE [21], EIGENSTRAT [10], ROADTRIPS [22], EMMAX [16], and TASSEL [17].

The PCA method and the mixed effects model are the most popular methods in genetic association studies. There are quite few comparisons regarding the performance of these two methods. However, these comparisons are almost all conducted via simulation studies [18, 23-25]. It is not clear whether the conclusions reached in such studies are applicable beyond the simulated situations. To avoid the limitations of simulation studies, we analytically compared the PCA method and the mixed effects model in terms of their validity and efficiency in testing the genetic effect in the presence of cryptic relatedness and population stratification, accompanied by a few examples of special situations and simulation studies.

In this paper, we first describe cryptic relatedness and population stratification in genetic association studies. We then introduce the PCA method and the mixed effects model method and compare them analytically first in the case of relatedness and then in the case of population stratification in two propositions. Under Validity and Efficiency of the Two Methods, we present some examples to illustrate the implication of these propositions, followed by further simulation studies in the Examples section to demonstrate our conclusions.

\section{Cryptic Relatedness and Population Stratification}

Let $y$ denote the value of a quantitative trait for a study subject. It is assumed that $y$ is continuous and is determined by the following additive polygenetic model [26]:

$$
y=\sum_{j} h_{j}+\varepsilon,
$$

where $h_{j}$ is the contribution of the $j$-th genetic factor to trait $y$ and $\varepsilon$ is the individual-specific error term. Under the additive polygenic model, the variance of $y$, denoted by $\sigma_{y}^{2}$, is the sum of the variance of the polygenic effects $\Sigma_{j} h_{j}$, denoted by $\sigma_{G}^{2}$, and the variance of the error term $\varepsilon$, denoted by $\sigma_{e}^{2}$. That is, $\sigma_{y}^{2}=\sigma_{G}^{2}+\sigma_{e}^{2}$.

In the presence of cryptic relatedness, the polygenic effects among the study subjects are correlated. Consequently, their trait values are also correlated. Let $\mathbf{y}=\left(y_{1}\right.$, $\left.y_{2}, \ldots, y_{n}\right)^{\prime}$ be a vector of trait values on $n$ study subjects. The correlation among subjects is often represented by a matrix of pair-wise relatedness, such as identical by state or Balding-Nichols similarity. Let $\mathbf{S}$ denote a matrix of pair-wise relatedness. So the variance of $\mathbf{y}$ is $\boldsymbol{\Omega}=\sigma_{G}^{2} \mathbf{S}+\sigma_{e}^{2} \mathbf{I}$. The mean of $\mathbf{y}$ is a vector of a constant and can be denoted by $u \mathbf{1}$ for a constant $u$, where $\mathbf{1}$ denotes a vector whose elements are equal to 1 .

If there exists population stratification, the polygenic effects are expected to be different in different subpopulations due to their allele frequency differences. Let $K$ be the total number of populations in the data, $u_{k}$ the mean trait value for the $k$-th population, $n_{k}$ the sample size from the $k$-th population, and $\mathbf{1}_{k}$ the $n_{k} \times 1$ vector of $1 \mathrm{~s}$, $k=1, \ldots, K$. Suppose that the subjects are organized in such a way that subjects from the same population are indexed next to each other. The mean trait value is $E(\mathbf{y})=$ $\mathbf{u}=\left(u_{1} \mathbf{1}_{1}^{\prime}, \ldots, u_{K} \mathbf{1}_{K}\right)^{\prime}$. The variance matrix of $\mathbf{y}$ given $\mathbf{u}$ remains $\boldsymbol{\Omega}=\sigma_{G}^{2} \mathbf{S}+\sigma_{e}^{2} \mathbf{I}$. That is, subjects are allowed to be related.

Let $\mathbf{g}$ be a $n \times 1$ vector of the genotype scores at the single nucleotide polymorphism being tested for association. Each component of $\mathbf{g}$ assumes a value 0,1 , or 2 . Let $\beta$ be the effect size of one unit change in the genotype score. We want to test for genetic association by testing the null hypothesis $H_{0}: \beta=0$ versus the alternative $H_{1}$ : $\beta \neq 0$.

For the purpose of this paper, it is assumed that if there is cryptic relatedness but not population stratification, the trait vector $\mathbf{y}$ is determined by

$$
\mathbf{y}=u \mathbf{1}+\beta \mathbf{g}+\varepsilon,
$$


where $\varepsilon \sim N(\mathbf{0}, \boldsymbol{\Omega})$. If there exists population stratification, the phenotype vector $y$ is assumed to come from the following model:

$$
\mathbf{y}=\mathbf{u}+\beta \mathbf{g}+\boldsymbol{\varepsilon},
$$

where $\varepsilon \sim N(\mathbf{0}, \boldsymbol{\Omega})$.

\section{The PCA Method and the Mixed Effects Model}

Given genotype vector $\mathbf{g}$, the variance matrix of $\mathbf{y}$ is different in equation 1 and 2 . In equation 1 , it is $\boldsymbol{\Omega}=\sigma_{G}^{2} \mathbf{S}$ $+\sigma_{e}^{2} \mathbf{I}$. In equation 2 , it is $\Omega^{*}$, which is defined as

$$
\begin{aligned}
\boldsymbol{\Omega}^{*} & =\operatorname{Var}(\mathbf{u}+\boldsymbol{\varepsilon}) \\
& =\sigma_{u}^{2} \tilde{\mathbf{S}}+\sigma_{G}^{2} \mathbf{S}+\sigma_{e}^{2} \mathbf{I},
\end{aligned}
$$

where $\sigma_{u}^{2}$ is the population variance in $u_{1}, u_{2}, \ldots, u_{K}$ and

$$
\tilde{\mathbf{S}}=\left(\begin{array}{llll}
\mathbf{1}_{1} \mathbf{1}_{1}^{\prime} & & & \\
& \mathbf{1}_{2} \mathbf{1}_{2}^{\prime} & & \\
& & \ddots & \\
& & & \mathbf{1}_{K} \mathbf{1}_{K}^{\prime}
\end{array}\right)
$$

is a relatedness matrix induced by population stratification. $\boldsymbol{\Omega}^{*}$ can be further written as

$$
\mathbf{\Omega}^{*}=\sigma_{G}^{2} \boldsymbol{S}^{*}+\sigma_{e}^{2} \mathbf{I},
$$

where $\sigma_{G^{\prime}}^{2}=\sigma_{u}^{2}+\sigma_{G}^{2}$ and $\mathbf{S}^{*}$ satisfies $\sigma_{G}^{2} \mathbf{S}^{*}=\sigma_{u}^{2} \tilde{\mathbf{S}}+\sigma_{G}^{2} \mathbf{S}$. $\mathbf{S}^{*}$ is the overall relatedness matrix.

Let $\mathbf{x}_{1}, \mathbf{x}_{2}, \ldots, \mathbf{x}_{m}$ denote the $m$ largest eigenvectors of matrix $\mathbf{S}$ or $\mathbf{S}^{*}$. In the PCA method, these eigenvectors are used as covariates in the following equation: $\mathbf{y}=\mathbf{Z} \boldsymbol{\alpha}+\beta \mathbf{g}$ $+\boldsymbol{\delta}$, where $\mathbf{Z}=\left(\mathbf{1}, \mathbf{X}_{m}\right)$ with $\mathbf{X}_{m}=\left(\mathbf{x}_{1}, \ldots, \mathbf{x}_{m}\right), \boldsymbol{\alpha}$ is a vector of regression coefficients, and $\boldsymbol{\delta}$ is a vector of independent identically normally distributed error terms. The leastsquares estimate of $\beta$ is

$$
\tilde{\beta}=\frac{\mathbf{g}^{\prime} \mathbf{P}_{1} \mathbf{y}}{\mathbf{g}^{\prime} \mathbf{P}_{1} \mathbf{g}},
$$

where $\mathbf{P}_{1}=\mathbf{I}-\mathbf{Z}\left(\mathbf{Z}^{\prime} \mathbf{Z}\right)^{-1} \mathbf{Z}^{\prime}$ is the projection matrix that projects a $n \times 1$ vector into a space orthogonal to the space spanned by the columns of matrix $\mathbf{Z}$.

The mixed effects model method does not include principal components as covariates. In the case of cryptic relatedness, it fits equation 1 assuming $\varepsilon \sim N(\mathbf{0}, \boldsymbol{\Omega})$. The generalized least-squares estimate of $\beta$ is

$$
\hat{\beta}=\frac{\mathbf{g}^{\prime} \mathbf{P}_{2} \mathbf{y}}{\mathbf{g}^{\prime} \mathbf{P}_{2} \mathbf{g}},
$$

where $\mathbf{P}_{2}=\boldsymbol{\Omega}^{-1}-\boldsymbol{\Omega}^{-1} \mathbf{1}\left(\mathbf{1}^{\prime} \mathbf{\Omega}^{-1} \mathbf{1}\right)^{-1} \mathbf{1}^{\prime} \mathbf{\Omega}^{-1}$ and $\mathbf{P}_{2}$ satisfies $\mathbf{P}_{2} \mathbf{1}=\mathbf{0}$. Noting that $\mathbf{P}_{2} \boldsymbol{\Omega} \mathbf{P}_{2}=\mathbf{P}_{2}$, the sampling variance of $\hat{\beta}$ is

$$
\operatorname{Var}(\hat{\beta})=\frac{1}{\mathbf{g}^{\prime} \mathbf{P}_{2} \mathbf{g}} .
$$

In the case of population stratification, $\Omega$ is replaced by $\Omega^{*}$.

\section{Validity and Efficiency of the Two Methods}

In this section, we compare analytically the PCA method and the mixed effects model method in terms of their validity and efficiency in the presence of cryptic relatedness and population stratification. We first consider the two methods when population stratification is absent and summarize the results in the following proposition.

\section{Proposition 1}

If there exists cryptic relatedness but not population stratification, both $\tilde{\beta}$ and $\hat{\beta}$ are unbiased estimates of $\beta$. However, the former has a larger variance than the latter does. That is, $\operatorname{Var}(\tilde{\beta}) \geq \operatorname{Var}(\hat{\beta})$. Consequently, the mixed effects model is more efficient.

It is easy to show that under equation 1 , the variance of $\tilde{\beta}$ is

$$
\operatorname{Var}(\tilde{\beta})=\frac{\mathbf{g}^{\prime} \mathbf{P}_{1} \mathbf{\Omega} \mathbf{P}_{1} \mathbf{g}}{\left(\mathbf{g}^{\prime} \mathbf{P}_{1} \mathbf{g}\right)^{2}} .
$$

The proof of unbiasedness is straightforward. When there is no population stratification, the phenotypic $\mathbf{y}$ follows equation 1 , and $E(\mathbf{y})=u \mathbf{1}+\beta \mathbf{g}$. Therefore

$$
\begin{aligned}
E(\tilde{\beta}) & =\left(\mathbf{g}^{\prime} \mathbf{P}_{1} \mathbf{g}\right)^{-1}\left[\mathbf{g}^{\prime} \mathbf{P}_{1} E(\mathbf{y})\right] \\
& =u\left(\mathbf{g}^{\prime} \mathbf{P}_{1} \mathbf{g}\right)^{-1} \mathbf{g}^{\prime} \mathbf{P}_{1} \mathbf{1}+\beta=\beta,
\end{aligned}
$$

and the PCA estimate $\tilde{\beta}$ is unbiased. Similarly, since $\mathbf{P}_{2} \mathbf{1}=$ $0, E(\hat{\beta})=\beta$ and $\hat{\beta}$ is unbiased.

Variance comparison between the two estimates can be carried out using the Cauchy-Schwarz inequality as follows. Define inner product $\left\langle\cdot\right.$, , . such that $\langle\mathbf{x}, \mathbf{y}\rangle=\mathbf{x}^{\prime} \mathbf{\Omega} \mathbf{y}$. Notice that $\mathbf{P}_{2} \boldsymbol{\Omega} \mathbf{P}_{2}=\mathbf{P}_{2}$ and $\mathbf{P}_{2} \boldsymbol{\Omega} \mathbf{P}_{1}=\left(\mathbf{I}-\boldsymbol{\Omega}^{-1} \mathbf{1}\left(\mathbf{1}^{\prime} \mathbf{\Omega}^{-1} \mathbf{1}\right)^{-1} \mathbf{1}^{\prime}\right)$ $\mathbf{P}_{1}=\mathbf{P}_{1}$, we have

$$
\begin{aligned}
\left(g^{\prime} \mathbf{P}_{2} g\right) \cdot\left(g^{\prime} \mathbf{P}_{1} \Omega \mathbf{P}_{1} \mathbf{g}\right) & =\left\langle\mathbf{P}_{2} \mathbf{g}, \mathbf{P}_{2} \mathbf{g}\right\rangle \cdot\left\langle\mathbf{P}_{1} \mathbf{g}, \mathbf{P}_{1} \mathbf{g}\right\rangle \\
& \geq\left(\left\langle\mathbf{P}_{2} \mathbf{g}, \mathbf{P}_{1} \mathbf{g}\right\rangle\right)^{2} \\
& =\left(\mathbf{g}^{\prime} \mathbf{P}_{2} \Omega \mathbf{P}_{1} \mathbf{g}\right)^{2} \\
& =\left(\mathbf{g}^{\prime} \mathbf{P}_{1} \mathbf{g}\right)^{2} .
\end{aligned}
$$


Given $\operatorname{Var}(\tilde{\beta})$ and $\operatorname{Var}(\hat{\beta})$ in equation 6 and 5 , it immediately follows that $\operatorname{Var}(\tilde{\beta}) \geq \operatorname{Var}(\hat{\beta})$. This completes the proof of Proposition 1.

It is a well-known textbook result that $\hat{\beta}$ is the best linear unbiased estimator of $\beta$ among generalized linear models and thus has the smallest variance [27, chapter 6]. Proposition 1 extends this result because there are more covariates available to the PCA method than to the mixed effects model. From the proof procedure one can see that no specific properties of $\left\{\mathbf{x}_{k}\right\}$ being eigenvectors are used. Proposition 1 remains true even when $\left\{\mathbf{x}_{k}\right\}$ are not eigenvectors.

When there is population stratification, we have the following results.

\section{Proposition 2}

In the presence of population stratification, we have (1) $E(\tilde{\beta})=\left(\mathbf{g}^{\prime} \mathbf{P}_{1} \mathbf{g}\right)^{-1}\left(\mathbf{g}^{\prime} \mathbf{P}_{1} \mathbf{u}\right)+\beta$.

That is, $\tilde{\beta}$ is a biased estimator of $\beta$ unless $\mathbf{u}$ satisfies $\mathbf{g}^{\prime} \mathbf{P}_{1} \mathbf{u}=0$. The bias is $E(\tilde{\beta})-\beta=\left(\mathbf{g}^{\prime} \mathbf{P}_{1} \mathbf{g}\right)^{-1}\left(\mathbf{g}^{\prime} \mathbf{P}_{1} \mathbf{u}\right)$.

(2) $E(\hat{\beta})=\left(\mathbf{g}^{\prime} \mathbf{P}_{2} \mathbf{g}\right)^{-1}\left(\mathbf{g}^{\prime} \mathbf{P}_{2} \mathbf{u}\right)+\beta$.

That is, $\beta$ is a biased estimator of $\beta$ unless $\mathbf{u}$ satisfies $\mathbf{g}^{\prime} \mathbf{P}_{2} \mathbf{u}=0$. The bias is $E(\hat{\beta})-\beta=\left(\mathbf{g}^{\prime} \mathbf{P}_{2} \mathbf{g}\right)^{-1}\left(\mathbf{g}^{\prime} \mathbf{P}_{2} \mathbf{u}\right)$.

The proof follows from the fact that $y$ follows equation 2 with $E(\mathbf{y})=\mathbf{u}+\beta \mathbf{g}$ when there is population stratification. The estimate of $\beta$ from the PCA method is $\tilde{\beta}=$ $\left(\mathbf{g}^{\prime} \mathbf{P}_{1} \mathbf{g}\right)^{-1} \mathbf{g}^{\prime} \mathbf{P}_{1} \mathbf{y}$, where $\mathbf{P}_{1}=\mathbf{I}-\mathbf{Z}\left(\mathbf{Z}^{\prime} \mathbf{Z}\right)^{-1} \mathbf{Z}^{\prime}, \mathbf{Z}=\left(1, \mathbf{X}_{m}\right)$, $\mathbf{X}_{m}=\left(\mathbf{x}_{1}, \ldots, \mathbf{x}_{m}\right)$, and $\mathbf{x}_{1}, \mathbf{x}_{2}, \ldots, \mathbf{x}_{m}$ are $m$ eigenvectors of $\mathbf{S}^{*}$ corresponding to its $m$ largest eigenvalues, and

$$
\begin{aligned}
E(\tilde{\beta}) & =\left(\mathbf{g}^{\prime} \mathbf{P}_{1} \mathbf{g}\right)^{-1} \mathbf{g}^{\prime} \mathbf{P}_{1} E(\mathbf{y}) \\
& =\left(\mathbf{g}^{\prime} \mathbf{P}_{1} \mathbf{g}\right)^{-1} \mathbf{g}^{\prime} \mathbf{P}_{1}(\mathbf{u}+\beta \mathbf{g}) \\
& =\left(\mathbf{g}^{\prime} \mathbf{P}_{1} \mathbf{g}\right)^{-1}\left(\mathbf{g}^{\prime} \mathbf{P}_{1} \mathbf{u}\right)+\beta .
\end{aligned}
$$

Similarly, the estimate of $\beta$ from the mixed effects model method is $\hat{\beta}=\left(\mathbf{g}^{\prime} \mathbf{P}_{2} \mathbf{g}\right)^{-1} \mathbf{g}^{\prime} \mathbf{P}_{2} \mathbf{y}$, where $\mathbf{P}_{2}=\mathbf{\Omega}^{*-1}-$ $\mathbf{\Omega}^{*-1} \mathbf{1}\left(\mathbf{1}^{\prime} \mathbf{\Omega}^{*-1} \mathbf{1}\right)^{-1} \mathbf{1}^{\prime} \mathbf{\Omega}^{*-1}$. This completes the proof.

Proposition 2 indicates that the estimate of $\beta$ from either method can be biased. The direction of the bias is determined by $\mathbf{g}^{\prime} \mathbf{P}_{1} \mathbf{u}$ and $\mathbf{g}^{\prime} \mathbf{P}_{2} \mathbf{u}$, respectively. This observation has two implications. First, if there is no genetic association (i.e. $\beta=0$ ), both methods are invalid. Second, if there is association, both methods can be conservative or anticonservative. The extent of which is affected by not only the direction of the bias in the estimate of $\beta$, but also the variance of the estimate of $\beta$. It is unclear which method has smaller bias in the estimate of $\beta$ or smaller variance of the estimate of $\beta$. We will investigate this issue more in the Examples section and in the Simulation Studies section.

\section{Examples}

\section{Example 1}

We first assume that the relatedness between individuals is the same for all pairs, which is defined as $\rho$, and there is no population stratification. That is, we consider equation 1 with

$$
\mathbf{S}=\left(\begin{array}{cccc}
1 & \rho & \ldots & \rho \\
\rho & 1 & \ldots & \rho \\
\vdots & \vdots & \vdots & \vdots \\
\rho & \rho & \ldots & 1
\end{array}\right)=(1-\rho) \mathbf{I}+\rho \mathbf{1 1}^{\prime}
$$

and $\boldsymbol{\Omega}=\sigma_{G}^{2} \mathbf{S}+\sigma_{e}^{2} \mathbf{I}=\theta^{2} \mathbf{I}+\rho \sigma_{G}^{2} \mathbf{1} \mathbf{1}^{\prime}$, where

$$
\theta=\sqrt{\sigma_{G}^{2}(1-\rho)+\sigma_{e}^{2}} .
$$

Since

$$
\begin{aligned}
\mathbf{\Omega}^{-1} & =\frac{1}{\theta^{2}}\left(\mathbf{I}-\frac{\rho \sigma_{G}^{2}}{\theta^{2}+n \rho \sigma_{G}^{2}} \mathbf{1}^{\prime}\right), \\
\mathbf{g}^{\prime} \mathbf{\Omega}^{-1} \mathbf{g} & =\frac{1}{\theta^{2}}\left(\mathbf{g}^{\prime} \mathbf{g}-\frac{\rho \sigma_{G}^{2}\left(\mathbf{1}^{\prime} \mathbf{g}\right)^{2}}{\theta^{2}+n \rho \sigma_{G}^{2}}\right), \\
\mathbf{1}^{\prime} \mathbf{\Omega}^{-1} \mathbf{g} & =\frac{1}{\theta^{2}}\left(1-\frac{n \rho \sigma_{G}^{2}}{\theta^{2}+n \rho \sigma_{G}^{2}}\right)\left(\mathbf{1}^{\prime} \mathbf{g}\right) \\
& =\frac{\mathbf{1}^{\prime} \mathbf{g}}{\theta^{2}+n \rho \sigma_{G}^{2}},
\end{aligned}
$$

and

$$
\mathbf{1}^{\prime} \mathbf{\Omega}^{-1} \mathbf{1}=\frac{n}{\theta^{2}}\left(1-\frac{n \rho \sigma_{G}^{2}}{\theta^{2}+n \rho \sigma_{G}^{2}}\right)=\frac{n}{\theta^{2}+n \rho \sigma_{G}^{2}},
$$

we have

$$
\begin{aligned}
{[\operatorname{Var}(\hat{\beta})]^{-1} } & =\mathbf{g}^{\prime} \mathbf{P} \mathbf{g} \\
& =\mathbf{g}^{\prime} \mathbf{\Omega}^{-1} \mathbf{g}-\frac{\left(\mathbf{1}^{\prime} \mathbf{\Omega}^{-1} \mathbf{g}\right)^{2}}{\mathbf{1}^{\prime} \mathbf{\Omega}^{-1} \mathbf{1}} \\
& =\frac{\mathbf{g}^{\prime} \mathbf{g}}{\theta^{2}}-\frac{\rho \sigma_{G}^{2}\left(\mathbf{1}^{\prime} \mathbf{g}\right)^{2}}{\theta^{2}\left(\theta^{2}+n \rho \sigma_{G}^{2}\right)}-\frac{\left(\mathbf{1}^{\prime} \mathbf{g}\right)^{2}}{n\left(\theta^{2}+n \rho \sigma_{G}^{2}\right)} \\
& =\frac{\mathbf{g}^{\prime} \mathbf{g}}{\theta^{2}}-\frac{\left(\mathbf{1}^{\prime} \mathbf{g}\right)^{2}}{n \theta^{2}} \\
& =\frac{n \sigma_{g}^{2}}{\theta^{2}}
\end{aligned}
$$

with $\sigma_{g}^{2}=n^{-1} \mathbf{g}^{\prime} \mathbf{g}-\left(n^{-1} \mathbf{1}^{\prime} \mathbf{g}\right)^{2}$. That ist, $\operatorname{Var}(\hat{\beta})=\theta^{2} / n \sigma_{g}^{2}$.

The variance of $\tilde{\beta}$ depends on the principal components included in the regression. The largest eigenvalue of matrix $\mathbf{S}$ is $\lambda_{1}=1+(n-1) \rho$ with associated eigenvector $\mathbf{x}_{1}=\left(n^{-1 / 2}, n^{-1 / 2}, n^{-1 / 2}\right)^{\prime}$. This eigenvector confounds with the intercept term and needs to be excluded. All other ei- 
genvalues are equal to $1-\rho$. The corresponding eigenvectors are not unique. For instance, one set of eigenvectors is

$$
\begin{aligned}
\mathbf{x}_{2}= & \left(\frac{1}{\sqrt{2}},-\frac{1}{\sqrt{2}}, 0, \ldots, 0\right)^{\prime} \\
& \vdots \\
\mathbf{x}_{i}= & \left(\frac{1}{\sqrt{(i-1) i}}, \ldots, \frac{1}{\sqrt{(i-1) i}},-\frac{i-1}{\sqrt{(i-1) i}}, 0, \ldots, 0\right)^{\prime} \\
& \vdots \\
\mathbf{x}_{n}= & \left(\frac{1}{\sqrt{(n-1) n}}, \ldots, \frac{1}{\sqrt{(n-1) n}},-\frac{n-1}{\sqrt{(n-1) n}}\right)^{\prime} .
\end{aligned}
$$

Switching any two elements in these vectors at the same time yields another set of eigenvectors.

Redefine $\mathbf{X}_{m}$ by $\mathbf{X}_{m}=\left(\mathbf{x}_{2}, \ldots, \mathbf{x}_{m}\right)$, the matrix formed by the first $m-1$ eigenvectors excluding $\mathbf{x}_{1}$. Since

$$
\mathbf{Z}^{\prime} \mathbf{Z}=\left(\begin{array}{cc}
n & \mathbf{1}^{\prime} \mathbf{X}_{m} \\
\mathbf{X}_{m}^{\prime} \mathbf{1} & \mathbf{X}_{m}^{\prime} \mathbf{X}_{m}
\end{array}\right)=\left(\begin{array}{cc}
n & 0 \\
0 & \mathbf{I}_{m}
\end{array}\right),
$$

the inverse of $\mathbf{Z}^{\prime} \mathbf{Z}$ is

$$
\left(\mathbf{Z}^{\prime} \mathbf{Z}\right)^{-1}=\left(\begin{array}{cc}
1 / n & 0 \\
0 & \mathbf{I}_{m}
\end{array}\right) .
$$

Therefore, $\mathbf{Z}\left(\mathbf{Z}^{\prime} \mathbf{Z}\right)^{-1} \mathbf{Z}^{\prime}=n^{-1} \mathbf{1} \mathbf{1}+\mathbf{X}_{m} \mathbf{X}_{m}^{\prime}$ and $\mathbf{P}_{1}=(\mathbf{I}-$ $\left.n^{-1} \mathbf{1} \mathbf{1}^{\prime}\right)-\mathbf{X}_{m} \mathbf{X}_{m}^{\prime}$. Since $\mathbf{P}_{1}$ is idempotent and $\mathbf{P}_{1} \mathbf{1}=\mathbf{0}$, we have $\mathbf{P}_{1} \mathbf{\Omega} \mathbf{P}_{1}=\theta^{2} \mathbf{P}_{1}$ and

$$
\operatorname{Var}(\tilde{\beta})=\frac{\mathbf{g}^{\prime} \mathbf{P}_{1} \mathbf{\Omega} \mathbf{P}_{1} \mathbf{g}}{\left(\mathbf{g}^{\prime} \mathbf{P} \mathbf{g}\right)^{2}}=\frac{\theta^{2}}{n \sigma_{g}^{2}-\left[\left(\mathbf{g}^{\prime} \mathbf{x}_{2}\right)^{2}+\ldots+\left(\mathbf{g}^{\prime} \mathbf{x}_{m}\right)^{2}\right]},
$$

where $\sigma_{g}^{2}=n^{-1} \mathbf{g}^{\prime} \mathbf{g}-\left(n^{-1} \mathbf{1} \mathbf{g}\right)^{2}$ is the variance of the components of vector $\mathbf{g}$. One can see that adding more eigenvectors increases the variance of $\tilde{\beta}$ unless the added eigenvectors are orthogonal to $\mathbf{g}$ (i.e. each of the added ones satisfies $\mathbf{g}^{\prime} \mathbf{x}_{m}=0$ ). In addition, for the same number of eigenvectors used (i.e. the value of $m$ is fixed), different choices of eigenvectors can result in a difference in efficiency. The ratio $\operatorname{Var}(\tilde{\beta}) / \operatorname{Var}(\hat{\beta})$ is

$$
\frac{\operatorname{Var}(\tilde{\beta})}{\operatorname{Var}(\hat{\beta})}=\frac{n \sigma_{g}^{2}}{\left(n \sigma_{g}^{2}-\mathbf{g}^{\prime} \mathbf{X}_{m} \mathbf{X}_{m}^{\prime} \mathbf{g}\right)} .
$$

This ratio does not depend on $\rho$. It is 1 if and only if $\mathbf{X}_{m}^{\prime} \mathbf{g}=0$. This happens if no principal component is used. This conclusion is very interesting in itself: although $\boldsymbol{\Omega}$ is

A Comparison of the PCA Method and the Mixed Effects Model not diagonal, the ordinary least-squares regression without using any principal components still performs better than the PCA method and as well as the mixed effects model. Another proof of this fact is to use existing results on the generalized linear squares theory. For instance, since

$$
\mathbf{\Omega} \cdot(\mathbf{1} \mathbf{g})=\left(\left(\theta^{2}+n \rho \sigma_{G}^{2}\right) \mathbf{1}, n \rho \bar{g} \sigma_{G}^{2} \mathbf{1}+\theta^{2} \mathbf{g}\right)=(\mathbf{1} \mathbf{g}) \cdot \mathbf{B},
$$

where

$$
\mathbf{B}=\left(\begin{array}{cc}
\theta^{2}+n \rho \sigma_{G}^{2} & n \rho \bar{g} \sigma_{G}^{2} \\
0 & \theta^{2}
\end{array}\right)
$$

is obviously nonsingular, it follows from theorem 6.1.1 by Amemiya [27] that, when no PCA is used, the variance of the estimate of $\beta$ based on the simple linear regression is the same as the variance of its generalized least-squares estimate.

\section{Example 2}

We consider a case with population stratification only, in which cryptic relatedness is absent. The vector $\mathbf{u}$ in equation 2 is $\mathbf{u}=\left(u_{1} \mathbf{1}_{1}{ }_{1}, \ldots, u_{K} \mathbf{1}_{K}{ }^{\prime}\right)^{\prime}$, and the variance of the elements of vector $\mathbf{u}$ is denoted by $\sigma_{u}^{2}$. Then we have $\Omega^{*}=$ $\sigma_{G}^{2} \mathbf{S}^{*}+\sigma_{e}^{2} \mathbf{I}=\sigma_{u}^{2} \tilde{\mathbf{S}}+\sigma_{e}^{2} \mathbf{I}$ with

$$
\tilde{\mathbf{S}}=\left(\begin{array}{llll}
\mathbf{1}_{1} \mathbf{1}_{1}^{\prime} & & & \\
& \mathbf{1}_{2} \mathbf{1}_{2}^{\prime} & & \\
& & \ddots & \\
& & & \mathbf{1}_{K} \mathbf{1}_{K}^{\prime}
\end{array}\right)
$$

being the relatedness matrix induced by population stratification.

To find $\tilde{\beta}$ from the PCA method, we consider eigenvectors from $\tilde{\mathbf{S}}$. The largest eigenvalue for $\mathbf{1}_{k} \mathbf{1}_{k}$ is $n_{k}$ with corresponding eigenvector $\mathbf{1}_{k}, k=1, \ldots, K$. All other eigenvalues are equal to 0 . It is easy to show that the eigenvalues of $\tilde{\mathbf{S}}$ consist of those of $\mathbf{1}_{k} \mathbf{1}_{k}^{\prime}, k=1, \ldots, K$. So the only nonzero eigenvalues are $n_{k}, k=1, \ldots, K$. It is straightforward to verify that the eigenvector corresponding to $n_{1}$ is $\left(\mathbf{1}_{1}^{\prime}, \mathbf{0}_{(-1)}^{\prime}\right)^{\prime}$, where the subscript $(-1)$ implies the part of the vector excluding the first $n_{1}$ elements. Eigenvectors corresponding to eigenvalues $n_{k}, k=2, \ldots, K$ can be similarly constructed.

Without loss of generality, assume that $n_{1}>n_{k}, k=2$, ..., $K$. The eigenvector associated with the largest eigenvalue of $\tilde{\mathbf{S}}$ is $\mathbf{x}_{1}=\left(\mathbf{1}_{1}^{\prime}, \mathbf{0}_{(-1)}^{\prime}\right)^{\prime}$. Using only this eigenvector in the PCA method (i.e. $m=1$ ), the matrix $\mathbf{P}_{1}$ turns out to be

$$
\mathbf{P}_{1}=\mathbf{I}-\left(\begin{array}{lll}
n_{1}^{-1} \mathbf{1}_{1} \mathbf{1}_{1}^{\prime} & \\
& \left(n-n_{1}\right)^{-1} \mathbf{1}_{(-1)} \mathbf{1}_{(-1)}^{\prime}
\end{array}\right) .
$$


Furthermore, $\mathbf{g}^{\prime} \mathbf{P}_{1} \mathbf{u}=\left(n-n_{1}\right) \operatorname{Cov}\left(\mathbf{u}_{(-1)}, \mathbf{g}_{(-1)}\right)$ and $\mathbf{g}^{\prime} \mathbf{P}_{1} \mathbf{g}=n_{1} \sigma_{g_{1}}^{2}+\left(n-n_{1}\right) \sigma_{g_{(-1)}}^{2}$, where

$$
\operatorname{Cov}\left(\mathbf{u}_{(-1)}, \mathbf{g}_{(-1)}\right)=\left(n-n_{1}\right)^{-1} \sum_{i \neq 1} n_{i} u_{i} \bar{g}_{i}-\left(n-n_{1}\right)^{-2}\left(\sum_{i \neq 1} n_{i} u_{i}\right) \cdot\left(\sum_{i \neq 1} n_{i} \bar{g}_{i}\right),
$$

$\sigma_{g_{1}}^{2}=n_{1}^{-1} \mathbf{g}_{1}^{\prime} \mathbf{g}_{1}-n_{1}^{-2}\left(\mathbf{g}_{1}^{\prime} \mathbf{1}_{1}\right)^{2}$, and $\sigma_{g_{(-1)}}^{2}=\left(n-n_{1}\right)^{-1} \mathbf{g}_{(-1)}^{\prime} \mathbf{g}_{(-1)}-$ $\left(n-n_{1}\right)^{-2}\left(\mathbf{g}_{(-1)}^{\prime} \mathbf{1}_{(-1)}\right)^{2}$. So the bias of $\tilde{\beta}$ is

$$
\frac{\mathbf{g}^{\prime} \mathbf{P}_{\mathbf{1}} \mathbf{u}}{\mathbf{g}^{\prime} \mathbf{P}_{1} \mathbf{g}}=\frac{\left(n-n_{1}\right) \operatorname{Cov}\left(\mathbf{u}_{(-1)}, \mathbf{g}_{(-1)}\right)}{n_{1} \sigma_{g_{1}}^{2}+\left(n-n_{1}\right) \sigma_{g_{(-1)}}^{2}} .
$$

This bias does not depend on $\sigma_{e}^{2}$. If there are only two populations (i.e. $K=2$ ), $\mathbf{u}_{(-1)}=u_{2} \mathbf{1}_{2}$ is a vector of constants and $\operatorname{Cov}\left(\mathbf{u}_{(-1)}, \mathbf{g}_{(-1)}\right)=0$. That is, $\tilde{\beta}$ is unbiased. Another explanation of this fact is that $\mathbf{u}$ is a linear combination of $\mathbf{1}$ and $\mathbf{x}_{1}$, two vectors contained in $\mathbf{Z}$.

For the mixed effects model's estimate of $\hat{\beta}$, we first note that the inverse of $\Omega^{*}$ is also block-diagonal with the $i$-th block equal to

$$
\mathbf{\Omega}_{i}^{*-1}=\frac{1}{\sigma_{e}^{2}}\left[\mathbf{I}_{i}-\frac{\sigma_{u}^{2} \alpha_{i}}{n_{i}} \mathbf{1}_{i} \mathbf{1}_{i}^{\prime}\right]
$$

where $\alpha_{i}=1 /\left(\sigma_{e}^{2} / n_{i}+\sigma_{u}^{2}\right)$. Since $\boldsymbol{\Omega}^{*-1} \mathbf{1}_{i}=n_{i}^{-1} \alpha_{i} \mathbf{1}_{i}$, we have

$$
\boldsymbol{\Omega}^{*-1} \mathbf{u}=\left(\begin{array}{c}
\left(u_{1} \alpha_{1} / n_{1}\right) \mathbf{1}_{1} \\
\left(u_{2} \alpha_{2} / n_{2}\right) \mathbf{1}_{2} \\
\vdots \\
\left(u_{K} \alpha_{K} / n_{K}\right) \mathbf{1}_{K}
\end{array}\right),
$$

$\mathbf{1}^{\prime} \mathbf{\Omega}^{*-1} \mathbf{u}=\sum_{k} \alpha_{k} u_{k}$, and $\mathbf{g}^{\prime} \mathbf{\Omega}^{*-1} \mathbf{u}=\sum_{k} \alpha_{k} u_{k} \bar{g}_{k}$. Furthermore, $\mathbf{1}_{i}^{\prime} \mathbf{\Omega}^{*-1} \mathbf{1}_{i}=\alpha_{i}$ and $\mathbf{g}^{\prime} \boldsymbol{\Omega}^{*-1} \mathbf{1}=\sum_{k} \alpha_{k} \bar{g}_{k}$. Hence

$$
\begin{aligned}
\mathbf{g}^{\prime} \mathbf{P}_{2} \mathbf{u} & =\mathbf{g}^{\prime}\left[\mathbf{\Omega}^{*-1}-\frac{\mathbf{\Omega}^{*-1} \mathbf{1} \mathbf{1}^{\prime} \mathbf{\Omega}^{*-1}}{\mathbf{1}^{\prime} \mathbf{\Omega}^{*-1} \mathbf{1}}\right] \mathbf{u} \\
& =\mathbf{g}^{\prime} \mathbf{\Omega}^{*-1} \mathbf{u}-\frac{\left(\mathbf{g}^{\prime} \mathbf{\Omega}^{*-1} \mathbf{1}\right)\left(\mathbf{1}^{\prime} \mathbf{\Omega}^{*-1} \mathbf{u}\right)}{\mathbf{1}^{\prime} \mathbf{\Omega}^{*-1} \mathbf{1}} \\
& =\sum_{k} \alpha_{k} u_{k} \bar{g}_{k}-\frac{\sum_{k} \alpha_{k} \bar{g}_{k} \sum_{k} \alpha_{k} u_{k}}{\sum_{k} \alpha_{k}} \\
& =\frac{\sum_{k \neq l} \alpha_{k} \alpha_{l} u_{k}\left(\bar{g}_{k}-\bar{g}_{l}\right)}{\sum_{k} \alpha_{k}} \\
& =\frac{\sum_{k<l} \alpha_{k} \alpha_{l}\left(u_{k}-u_{l}\right)\left(\bar{g}_{k}-\bar{g}_{l}\right)}{\sum_{k} \alpha_{k}},
\end{aligned}
$$

where $\bar{g}_{k}$ is the average of the genotype score for population $k$ and $\sigma_{g_{k}}^{2}$ is defined in the same way as $\sigma_{g_{1}}^{2}$. Similarly,

$$
\mathbf{g}^{\prime} \mathbf{P}_{2} \mathbf{g}=\frac{1}{\sigma_{e}^{2}} \sum_{k} n_{k} \sigma_{g_{k}}^{2}+\frac{\sum_{k<l} \alpha_{k} \alpha_{l}\left(\bar{g}_{k}-\bar{g}_{l}\right)^{2}}{\sum_{k} \alpha_{k}} .
$$

From these results one would be able to compute the bias $\left(\mathbf{g}^{\prime} \mathbf{P}_{2} \mathbf{u}\right) /\left(\mathbf{g}^{\prime} \mathbf{P}_{2} \mathbf{g}\right)$ for the estimate of $\hat{\beta}$.

\section{Simulation Studies}

The intension of this section is not to provide largescale simulation studies. Such studies have been conducted previously elsewhere [18, 23-25]. The goal of our simulation studies is to demonstrate numerically the examples in the previous section. We generated data from either equation 1 or 2 . The matrix $\Omega$ is fixed, and the number of simulation replications in each study is 1,000 .

The first simulation study corresponds to Example 1 with $u=1, \rho=0.01, \sigma_{G}^{2}=1$, and $\sigma_{e}^{2}=1$. There are 500 subjects in each sample. The genotype score is 0 for the first 50, 1 for the next 150, and 2 for the last 300. In the PCA method, we consider the first two principal components from $\mathbf{S}$. Since $\mathbf{x}_{1}=\mathbf{1}$, this mounts to choose $\mathbf{x}_{2}$. We choose $\mathbf{x}_{2}$ to be proportional to a vector whose first 200 elements are equal to 1 , the next 200 are equal to -1 , and the last 100 are equal to 0 . The ratio $\operatorname{Var}(\tilde{\beta}) / \operatorname{Var}(\hat{\beta})$ is equal to $n \sigma_{\mathrm{g}}^{2} /$ $\left(n \sigma_{\mathrm{g}}^{2}-\left(\mathbf{g}^{\prime} \mathbf{x}_{2}\right)^{2}\right)=n \sigma_{\mathrm{g}}^{2} /\left(n \sigma_{\mathrm{g}}^{2}-(-12.5)^{2}\right)=3.252$. The choice of $\mathbf{x}_{2}$ has a great impact on the variance of $\tilde{\beta}$. For example, if $\mathbf{x}_{2}$ is chosen to be a vector whose first element is $1 / \sqrt{ } 2$, the last element $-1 / \sqrt{ } 2$, and all other elements 0 , the ratio $\operatorname{Var}(\tilde{\beta}) / \operatorname{Var}(\hat{\beta})$ would be merely 1.009 . Figure 1 presents the result with $\beta=0$. It shows that $\tilde{\beta}$ has larger variance, and the distributions of the squared $\mathrm{t}$ statistic from both methods conform with the 1-d.f. $\chi^{2}$ distribution. To investigate the power loss due to the use of principal components, we also consider the case where $\beta=0.1$ (fig. 2). $\operatorname{Var}(\tilde{\beta})$ continues to have larger variance. The mixed effects model is more powerful, as indicated by its tendency of having larger (squared) t statistic.

The second simulation study corresponds to Example 2 with $K=4$. Accordingly, the vector $\mathbf{u}$ has four segments, with one segment corresponding to one population. Genotype counts in each population and the value of $\mathbf{u}$ in the population are shown in table 1 . Hence the matrix $\boldsymbol{\Omega}=$ $\sigma_{u}^{2} \tilde{\mathbf{S}}+\mathbf{I}$, where

$$
\tilde{\mathbf{S}}=\left(\begin{array}{llll}
\mathbf{1}_{1} \mathbf{1}_{1}^{\prime} & & & \\
& \mathbf{1}_{2} \mathbf{1}_{2}^{\prime} & & \\
& & \mathbf{1}_{3} \mathbf{1}_{3}^{\prime} & \\
& & & \mathbf{1}_{4} \mathbf{1}_{4}^{\prime}
\end{array}\right) \text {. }
$$

We first investigate the unbiasedness of the estimates from the PCA method and the mixed effects model method when the genetic effect does not exist (i.e. $\beta=0$ ). We first used the first two principal components of $\tilde{\boldsymbol{S}}$ in the PCA method (fig. 3) and then the first four principal components (fig. 4). The first two principal components are indicator vectors for population 1 and population 4 , the popu- 


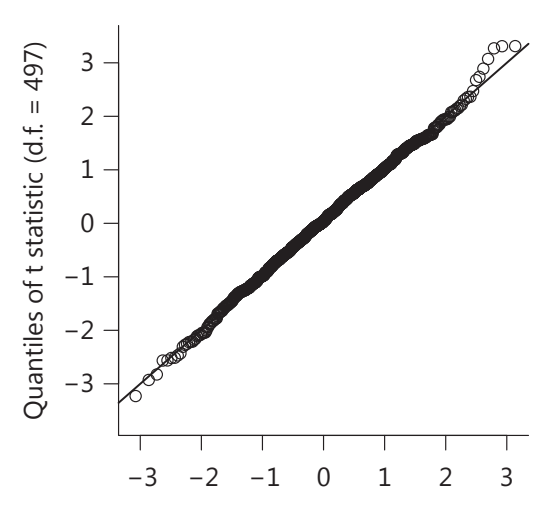

a Quantiles of t distribution (d.f. $=497$ )

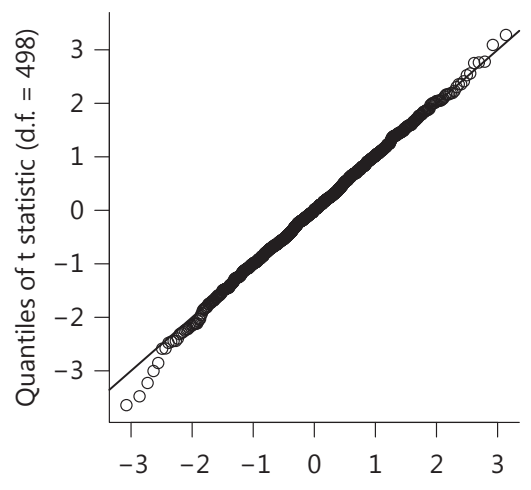

b

Quantiles of t distribution (d.f. $=498$ )

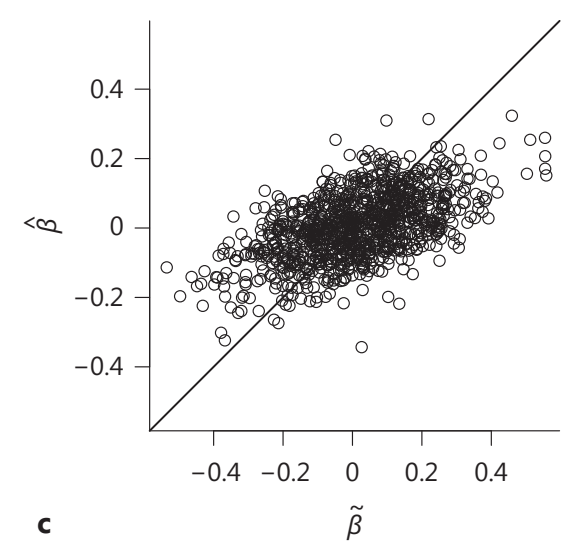

Fig. 1. Simulation results for the case of one population. The first two principal components are used for the PCA method. The genetic effect is $\beta=0$. $\mathbf{a}, \mathbf{b}$ QQ plot using the PCA method. c Comparison of $\tilde{\beta}$ versus $\hat{\beta}$.

Fig. 2. Simulation results for the case of one population. The first two principal components are used for the PCA method. The genetic effect is $\beta=0.1$. a Comparison of $\mathrm{t}$ statistic. b Comparison of $\tilde{\beta}$ versus $\hat{\beta}$.

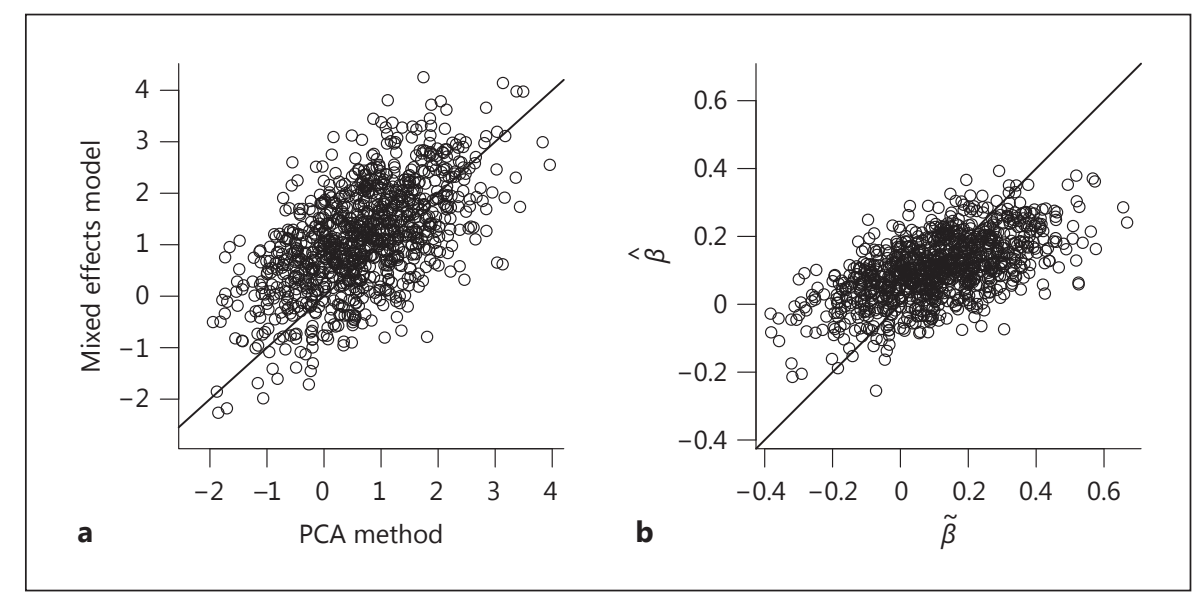

lations have the largest number of subjects. The first four principal components are indicator vectors for each of the four populations. Due to their collinearity with the regression intercept, using four of them is equivalent to using any three of them.

The PCA method in figure 3 is clearly invalid. Since the rank of $\tilde{\mathbf{S}}$ is 4 , there is still some population structure unexplained by the first two principal components. The PCA method treats the residuals as independent and identically distributed, while they are actually not. Bias in $\tilde{\beta}$ and inflation of the squared $t$ statistic are expected. However, when four principal components are used, the bias in $\tilde{\beta}$ is substantially reduced. In figures 3 and 4 , there is no change to the mixed effects model method.
Table 1. Genotype counts and the value of $\mathbf{u}$ in each population for the simulation study involving four populations

\begin{tabular}{llrrrl}
\hline Population & $u_{\mathrm{i}}$ & \multicolumn{5}{l}{ Genotype score } \\
\cline { 3 - 6 } & & 0 & 1 & 2 & total $\left(n_{\mathrm{i}}\right)$ \\
\hline 1 & 1 & 240 & 30 & 30 & 300 \\
2 & 2 & 20 & 160 & 20 & 200 \\
3 & 3 & 20 & 70 & 60 & 150 \\
4 & 4 & 70 & 70 & 160 & 300 \\
\hline
\end{tabular}




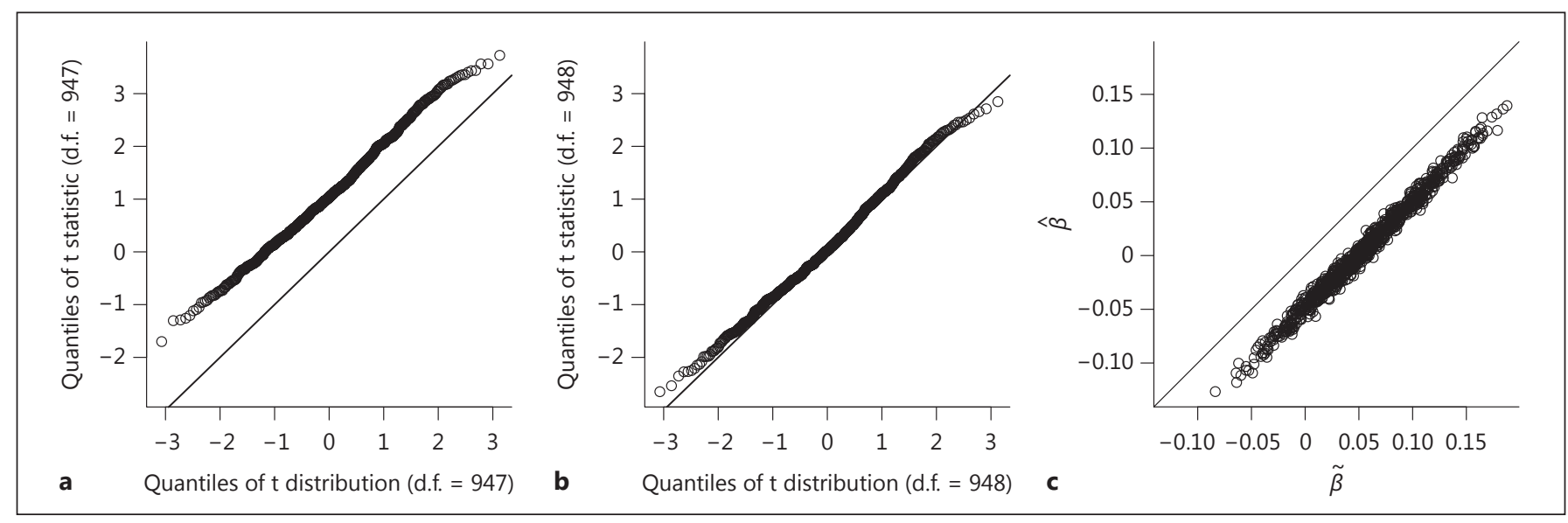

Fig. 3. Simulation results for the case of four populations. The first two principal components are used in the PCA method. Genetic effect size is $\beta=0$. $\mathbf{a}, \mathbf{b}$ QQ plot using the PCA method. c Comparison of $\tilde{\beta}$ versus $\hat{\beta}$.

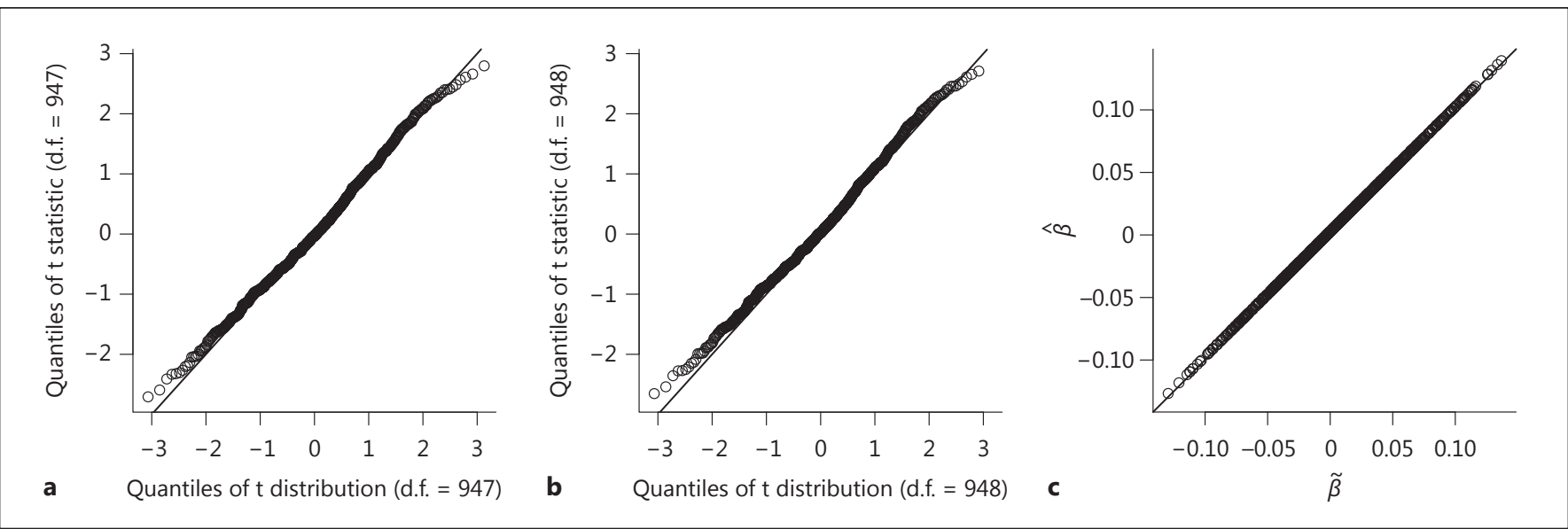

Fig. 4. Simulation results for the case of four populations. The first four principal components are used in the PCA method. Genetic effect size is $\beta=0$. $\mathbf{a}, \mathbf{b}$ QQ plot using the PCA method. c Comparison of $\tilde{\beta}$ versus $\hat{\beta}$.

\section{Discussion}

Large-scale genetic association studies is a popular means for identifying genetic factors underlying complex human traits. Cryptic relatedness and population stratification are deemed to be issues unavoidable in such studies. In this work, we have conducted an analytical comparison of two popular methods, i.e. the PCA method and the mixed effects model, that are supposed to address these issues. We focused not only on the efficiency of each method, but also on their validity through investigation of the bias and the variance of genetic effect estimates. The findings are enlightening.
If there exists cryptic relatedness but not population stratification, the mixed effects model is preferred as it has overall better performance. When there is no association, both methods are valid. However, if there is association, the mixed effects model is more powerful (Proposition 1). If there exists population stratification, both methods can lead to biased estimates of the genetic effect (Proposition 2). In other words, none of them can eliminate the confounding effect of population stratification. However, simulation studies suggest that the mixed effects model is much less affected by population stratification (fig. 3) and performs similarly to the PCA method when the population stratification is completely absent 
(fig. 4). These findings are consistent with simulation studies reported previously $[18,23]$.

The eigenvectors are useful in graphically representing population stratification. But the PCA method is generally not better than the mixed effects model in controlling for the confounding effect of population stratification for reasons discussed previously. In addition, our research reveals some issues not discussed before in using the PCA method. Previous research has focused on the number of principal components to use. The issue of what specific components to use appears to be ignored. Our research indicates that, if the relatedness is constant for any pairs (Example 1), different choices of the eigenvectors will affect the bias of the genetic effect estimate and efficiency in association testing. This impact can even be larger than the impact of the choice of the number of eigenvectors (Example 1).
We focused on continuous traits. When the trait is dichotomous, a common approach is to code the trait values as $0 \mathrm{~s}$ and $1 \mathrm{~s}$ and treat them as if they were continuous [see, for instance, 16, 22, 28]. This approach is asymptotically equivalent to the test based on a logistic regression. The conclusion of Proposition 1 and Proposition 2 applies to such recoded binary trait values.

\section{Acknowledgements}

The authors would like to thank an associate editor and two reviewers for their helpful comments. The work of Yingwei Peng is supported in part by a research grant from the Natural Sciences and Engineering Research Council of Canada.

\section{References}

-1 Campbell CD, Ogburn EL, Lunetta KL, Lyon HN, Freedman ML, Groop LC, Altshuler D, et al: Demonstrating stratification in a European American population. Nat Genet 2005; 37:868-872.

$\checkmark 2$ Xu S, Yin X, Li S, Jin W, Lou H, Yang L, Gong $\mathrm{X}$, et al: Genomic dissection of population substructure of Han Chinese and its implication in association studies. Am J Hum Genet 2009;85:762-774.

3 Chen J, Zheng H, Bei JX, Sun L, Jia WH, Li T, Zhang F, et al: Genetic structure of the Han Chinese population revealed by genome-wide SNP variation. Am J Hum Genet 2009;85: 775-785.

4 Cornelis MC, Agrawal A, Cole JW, Hansel $\mathrm{NH}$, Barnes KC, Beaty TH, Bennett SN, et al: The gene, Environment Association Studies consortium (GENEVA): maximizing the knowledge obtained from GWAS by collaboration across studies of multiple conditions. Genet Epidemiol 2010;34:364-372.

5 Devlin B, Roeder K: Genomic control for association studies. Biometrics 1999;55:9971004.

6 Bacanu S, Devlin B, Roeder K: The power of genomic control. Am J Hum Genet 2000;66: 1933-1944.

7 Wang K: Testing for genetic association in the presence of population stratification in genome-wide association studies. Genet Epidemiol 2009;33:637-645.

8 Pritchard JK, Rosenberg NA: Use of unlinked genetic markers to detect population stratification in association studies. Am J Hum Genet 1999;65:220-228.

9 Pritchard JK, Stephens M, Rosenberg NA, Donnelly P: Association mapping in structured populations. Am J Hum Genet 2000;67: 170-181.
10 Price AL, Patterson NJ, Plenge RM, Weinblatt ME, Shadick NA, Reich D: Principal components analysis corrects for stratification in genome-wide association studies. Nat Genet 2006;38:904-909.

11 Epstein MP, Allen AS, Satten GA: A simple and improved correction for population stratification in case-control studies. Am J Hum Genet 2007;80:921-930.

12 Balding DJ, Nichols RA: A method for quantifying differentiation between populations at multi-allelic loci and its implications for investigating identity and paternity. Genetica 1995;96:3-12.

13 Yang J, Benyamin B, McEvoy BP, Gordon S, Henders AK, Nyholt DR, Madden PA, et al: Common SNPs explain a large proportion of the heritability for human height. Nat Genet 2010;42:565-569.

14 Powell JE, Visscher PM, Goddard ME: Reconciling the analysis of IBD and IBS in complex trait studies. Nat Rev Genet 2010;11:800-805.

15 Yang J, Lee SH, Goddard ME, Visscher PM: GCTA: a tool for genome-wide complex trait analysis. Am J Hum Genet 2011;88:76-82.

16 Kang HM, Sul JH, Service SK, Zaitlen NA, Kong SY, Freimer NB, Sabatti C, et al: Variance component model to account for sample structure in genome-wide association studies. Nat Genet 2010;42:348-354.

17 Zhang Z, Ersoz E, Lai CQ, Todhunter RJ, Tiwari HK, Gore MA, Bradbury PJ, et al: Mixed linear model approach adapted for genomewide association studies. Nat Genet 2010;42: 355-360.

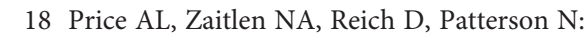
New approaches to population stratification in genome-wide association studies. Nat Rev Genet 2010;11:459-463.
19 Pritchard JK, Stephens M, Donnelly P: Inference of population structure using multilocus genotype data. Genetics 2000;155:945-959.

20 Rosenberg NA, Pritchard JK, Weber JL, Cann HM, Kidd KK, Zhivotovsky LA, Feldman MW: Genetic structure of human populations. Science 2002;298:2381-2385.

21 Alexander D, Novembre J, Lange K: Fast model-based estimation of ancestry in unrelated individuals. Genome Res 2009;19:16551664.

22 Thornton T, McPeek MS: ROADTRIPS: casecontrol association testing with partially or completely unknown population and pedigree structure. Am J Hum Genet 2010;86: 172-184.

23 Wu C, DeWan A, Hoh J, Wang Z: A comparison of association methods correcting for population stratification in case-control studies. Ann Hum Genet 2011;75:418-427.

24 Bouaziz M, Ambroise C, Guedj M: Accounting for population stratification in practice: a comparison of the main strategies dedicated to genome-wide association studies. PLoS One 2011;6:e28845.

25 Liu N, Zhao H, Patki A, Limdi NA, Allison DB: Controlling population structure in human genetic association studies with samples of unrelated individuals. Stat Interface 2011; 4:317-326.

26 Lange K: Mathematical and Statistical Methods for Genetic Analysis, ed 2. New York, Springer, 2002.

27 Amemiya T: Advanced Econometrics. Cambridge, Harvard University Press, 1985.

28 Rakovski CS, Stram DO: A kinship-based modification of the Armitage trend test to address hidden population structure and small differential genotyping errors. PLoS One 2009;4:e5825.
A Comparison of the PCA Method and the Mixed Effects Model
Hum Hered 2013;76:1-9

DOI: $10.1159 / 000353345$ 